The INL is a

U.S. Department of Energy

National Laboratory

operated by

Battelle Energy Alliance

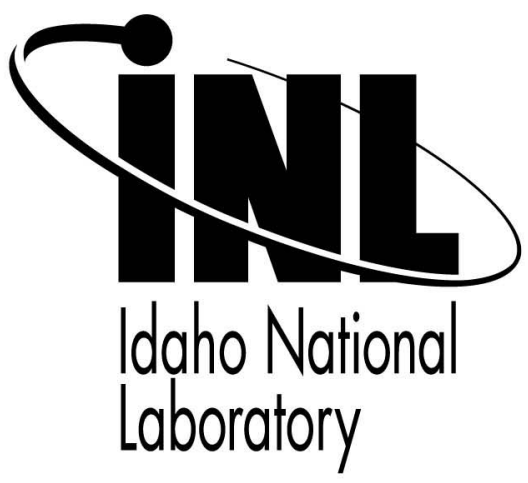

INL/CON-09-15212

PREPRINT

\title{
Imaging Carrier and Phonon Transport in Si Using Ultrashort Optical Pulses
}

\section{Photonics West -- OPTO}

\author{
D. H. Hurley \\ O. B. Wright \\ O. Matsuda \\ Brian E. McCandless \\ Subhash Shinde
}

January 2009

This is a preprint of a paper intended for publication in a journal or proceedings. Since changes may be made before publication, this preprint should not be cited or reproduced without permission of the author. This document was prepared as an account of work sponsored by an agency of the United States Government. Neither the United States Government nor any agency thereof, or any of their employees, makes any warranty, expressed or implied, or assumes any legal liability or responsibility for any third party's use, or the results of such use, of any information, apparatus, product or process disclosed in this report, or represents that its use by such third party would not infringe privately owned rights. The views expressed in this paper are not necessarily those of the United States Government or the sponsoring agency. 


\title{
Imaging carrier and phonon transport in Si using ultrashort optical pulses
}

\author{
D. H. Hurley ${ }^{\mathrm{a}^{*}}$, O. B. Wright ${ }^{\mathrm{b}}$, O. Matsuda ${ }^{\mathrm{b}}$, Brian E. McCandless ${ }^{\mathrm{c}}$, Subhash Shinde ${ }^{\mathrm{d}}$ \\ ${ }^{a}$ Idaho National Laboratory, P.O. Box 1625, Idaho Falls, Idaho 83415-2209, USA \\ ${ }^{\mathrm{b}}$ Department of Applied Physics, Hokkaido University, Sapporo 060-8628, Japan \\ 'Institute of Energy Conversion, University of Delaware, Newark, DE 19716 USA \\ ${ }^{\mathrm{d}}$ Sandia National Laboratory, P.O. Box 5800, Albuquerque, NM 87185-0603, USA
}

\begin{abstract}
A series of experiments have been conducted that microscopically image thermal diffusion and surface acoustic phonon propagation within a single crystallite of a polycrystalline Si sample. The experimental approach employs ultrashort optical pulses to generate an electron-hole plasma and a second probe pulse is used to image the evolution of the plasma. By decomposing the signal into a component that varies with delay time and a steady state component that varies with pump modulation frequency, the respective influence of carrier recombination and thermal diffusion are identified. Additionally, the coherent surface acoustic phonon component to the signal is imaged using a Sagnac interferometer to monitor optical phase.
\end{abstract}

Keywords: Thermal transport, phonon, electron transport, recombination, surface acoustic wave, grain boundary

\section{INTRODUCTION}

Studies of ultrafast optical excitation and propagation of phonon pulses hold the potential to unlock fundamental carrier and phonon transport properties of solar electric and optoelectronic materials. This approach, termed picosecond acoustics $^{1}$, is a valuable tool to study carrier ${ }^{2}$ and phonon transport ${ }^{1}$, since phonon generation and propagation are indicative of carrier relaxation ${ }^{3}$, carrier diffusion ${ }^{4}$, carrier-phonon interaction ${ }^{5}$ and phonon diffusion ${ }^{6}$. In the present study ultrashort optical pulses are used to generate an electron-hole plasma in a Si polycrystal and a second probe pulse is used to microscopically image evolution of the plasma and heat diffusion within a single crystallite. Additionally, the coherent surface acoustic phonon component to the signal is imaged using a Sagnac interferometer to monitor optical phase.

The sample is a Boron doped (p-type) Si polycrystal with a doping concentration of $2 \times 10^{16} / \mathrm{cm}^{3}$. The sample surface was mechanically polished without subsequent surface passivation resulting in a large density of surface defects. An electron backscatter micrograph of the sample is shown in the inset of Fig. 1. This micrograph gives the crystallite orientation in false color. The region of interest, which is removed from any grain boundaries, is denoted by the dashed square in the inset. The orientation of this crystallite is specified by a crystal plane perpendicular to the sample normal $(\mathrm{i}, \mathrm{j}, \mathrm{k})=(6,7,6)$ and a crystal direction parallel to the laboratory y axis $[\mathrm{u}, \mathrm{v}, \mathrm{w}]=[-7,-12,21]$. The present investigation is to serve as a baseline study from which to compare future studies that investigate the influence of boundaries (e.g. grain boundaries and bicrystal interfaces) on phonon transport.

\subsection{Experimental setup}

The experimental setup used for imaging changes in reflectivity and optical phase is shown in Fig. 1. It is similar to the setup presented by Tachizaki et al..$^{7}$ and involves translation of a probe beam relative to a fixed pump beam. The

*Email: david.hurley@inl.gov 


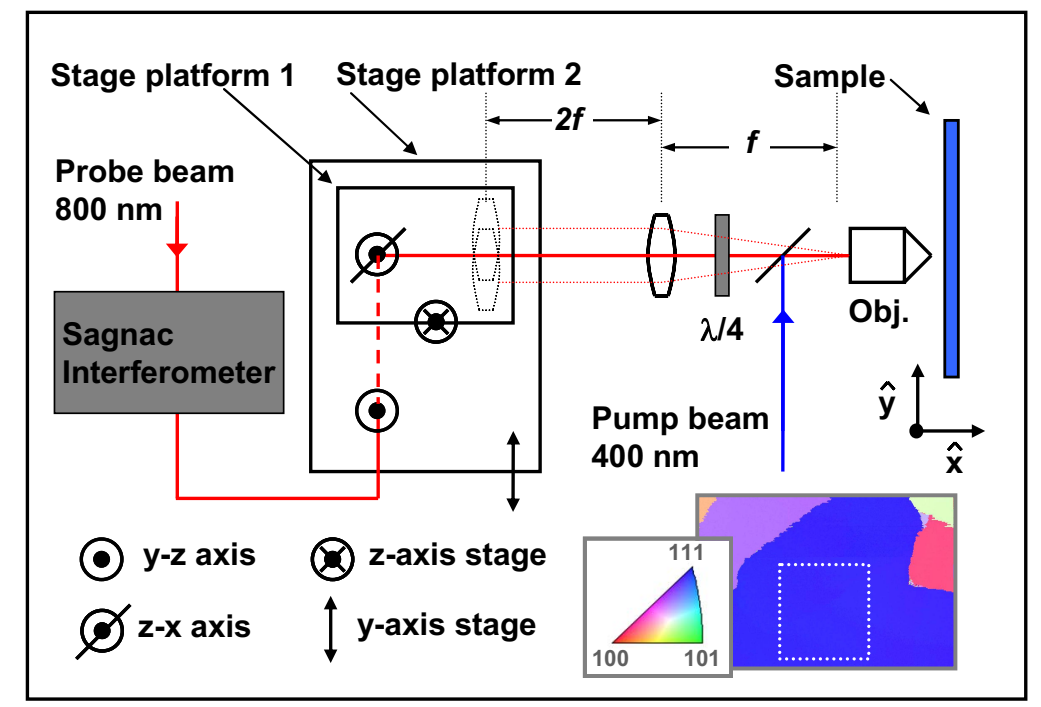

Fig. 1. Experimental setup. A single probe or two interferometric beams can be used. The probe beam is scanned relative to the pump beam at the focal point of the microscope objective by using the translation stage telescope combination.

experimental apparatus can employ either a single probe pulse for monitoring changes in probe reflectivity or two interferometric probe pulses for monitoring changes in optical phase and reflectivity. The probe is then sent through a telescope and focused onto the sample using a microscope objective. Both lenses of the telescope have a $100 \mathrm{~mm}$ focal length. The first lens is attached to a stage system that allows the lens and the probe beam to be translated in the $\mathrm{y}-\mathrm{z}$ plane (the probe beam propagation vector remains collinear with the lens axis). The second lens serves to converts the $y-z$ motion of the beam into a change in entrance angle into the objective. Since the objective is placed at the focal point of the second lens, the entrance angle can be changed without translation in the $y-z$ plane. The pump beam is guided along the optical axis of the objective using a dichroic beam splitter place after the telescope. This system provides a convenient means to scan the probe beam relative to the pump beam for situations that allow for only single-sided access.

\subsection{Results}

The first experiment involves imaging changes in probe reflectivity for different pump probe delay times. The pump was modulated at $100 \mathrm{kHz}$ to aid in lock-in detection. The pump $(\lambda=400 \mathrm{~nm}$, pulse energy $=0.026 \mathrm{~nJ})$ and probe $(\lambda=800$ $\mathrm{m}$, pulse energy $=0.013 \mathrm{~nJ}$ ) are derived from a Ti:Sapphire laser with a pulse duration $\sim 1 \mathrm{ps}$. The series of images in Fig. 2 gives the lock-in amplitude (top) and lock-in phase (bottom) of the change in reflectivity at 3 delays times: $t=0 \mathrm{ps,}$ $t=50 \mathrm{ps}$ and $t=100 \mathrm{ps}$.

To better understand the evolution of these images, it is helpful to decompose the signal into two components. The first component is broad band and varies with delay time (transient). The second component is narrow band and varies with pump modulation frequency (diffusion wave). Both components are driven by the diffusion of heat and photoexcited carriers. Initially $(t=0 \mathrm{ps}$ and $t=50 \mathrm{ps})$ the transient components to the signal are dominant. To illustrate this point consider the lock-in phase signal at these early times. There is little lateral variation in the phase signal for an area that encompasses the excitation region. Initially, the primary gradient in temperature and carrier density is in a direction perpendicular to the surface (pump skin depth=82 nm). Thus any lock-in phase variation of the transient signal would be in a direction perpendicular to the surface. Moreover, the reflectivity change of the probe is a phase average response over a distance equal to approximately one probe skin depth $(10 \mu \mathrm{m})$ which is considerably larger than the thermal diffusion length $\left(D_{t} t\right)^{1 / 2}$ and the plasma diffusion length $\left(D_{e} t\right)^{1 / 2}$ at these early times $\left(D_{t}\right.$ is the thermal diffusivity, $D_{e}$ is the ambipolar diffusivity and $t$ is the pump delay time). 

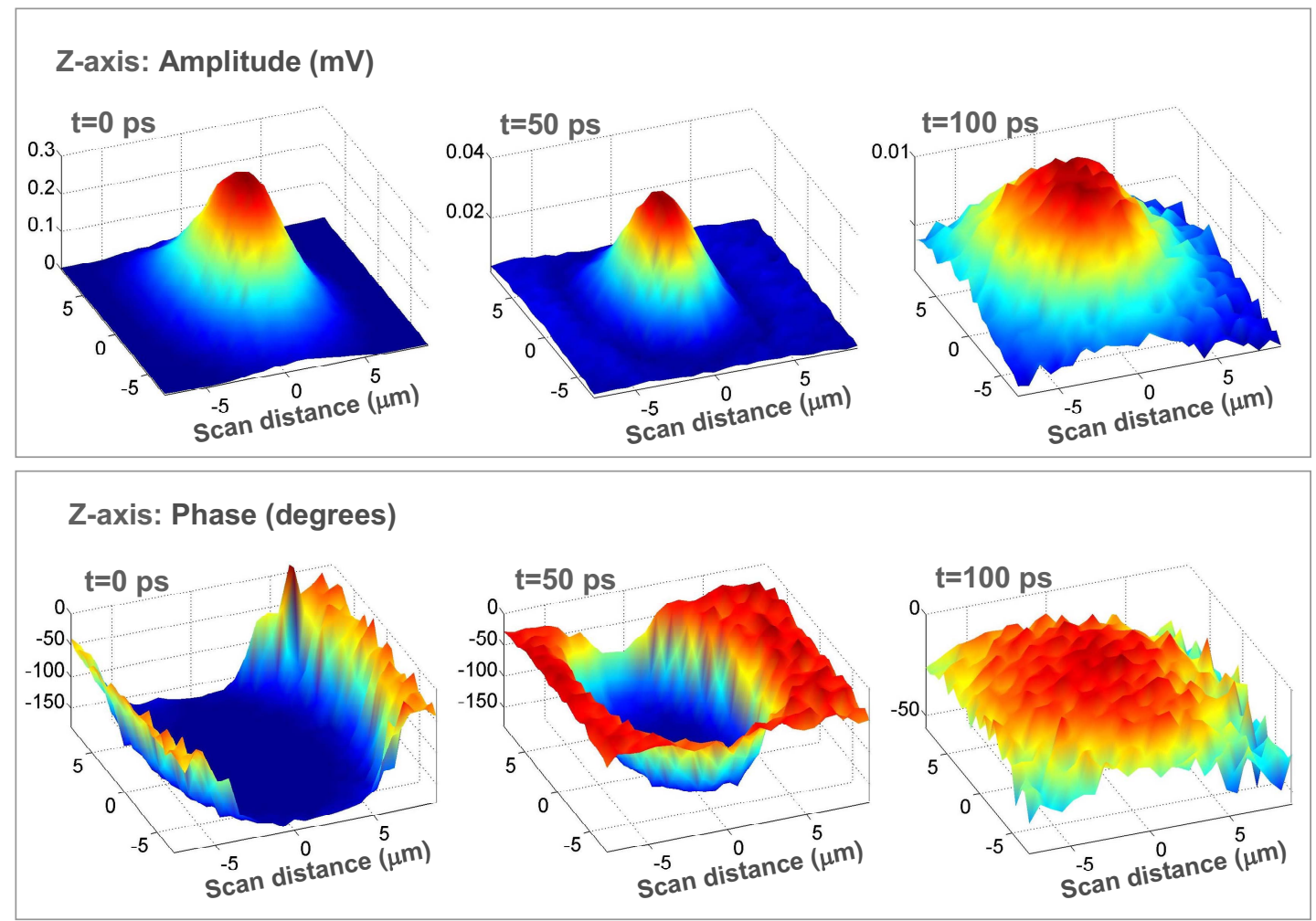

FIG. 2. Images of the amplitude and phase of the probe reflectivity change as a function of delay time. At early times the transient component that varies with delay time is dominant while at large delay times the steady state diffusion wave component is dominant.

The lock-in amplitude and phase images at 100 ps show the characteristics of a diffusion wave. In this case the lock-in phase and amplitude are associated with the amplitude and phase lag of the diffusion wave. Unlike the previous phase signals, the phase lag linearly decreases with increasing distance from the source (a constant was added to all of the phase data so as to give a maximum value of zero at $\mathrm{t}=100 \mathrm{ps}$ ). Moreover, the full width at half maximum (FWHM) of the amplitude signal at $100 \mathrm{ps}$ is considerably larger than the FWHM of the previous amplitude signals. In Fig. 3a, the FWHM of the central region of the amplitude signal as a function of delay time is shown. The FWHM continues to decrease up to approximately $80 \mathrm{ps}$. After this time the FWHM suddenly jumps up to a value of approximately $10 \mu \mathrm{m}$ and appears to remain constant with increasing delay. Continuing to accurately map the FWHM with increasing delay raises concern regarding slight defocusing of the laser spot due to beam divergence. This issue is conveniently circumvented by looking at $t=-10 \mathrm{ps}$ which is equivalent to $t=13.1 \mathrm{~ns}$ (the inverse of the laser repetition frequency). This enables a large temporal scan without a large spatial scan. A comparison between the normalized amplitude profile at $t=0 \mathrm{ps}$ and $t=13.1 \mathrm{~ns}$ is shown in Fig. 3b. The FWHM at $t=13.1 \mathrm{~ns}$ is $10.7 \mu \mathrm{m}$ which compares closely with the FWHM values after a delay of 90 ps. Why the FWHM steadily decreases ${ }^{4}$ at early times can be understood by considering the addition of two Gaussian profiles: one representing the transient component and one representing the steady state diffusion wave component. The lock-in phase data presented in Fig. 2 shows that these two components are $\sim 180$ degrees out of phase. Thus the functional form of the amplitude profile for $\mathrm{z}=0$ is approximated by the following:

$$
\frac{\Delta R}{R}=\left|A \exp \left(\frac{-y^{2}}{a_{t}^{2} / 4 \ln (2)}\right)-\exp \left(\frac{-y^{2}}{a_{s}^{2} / 4 \ln (2)}\right)\right|
$$



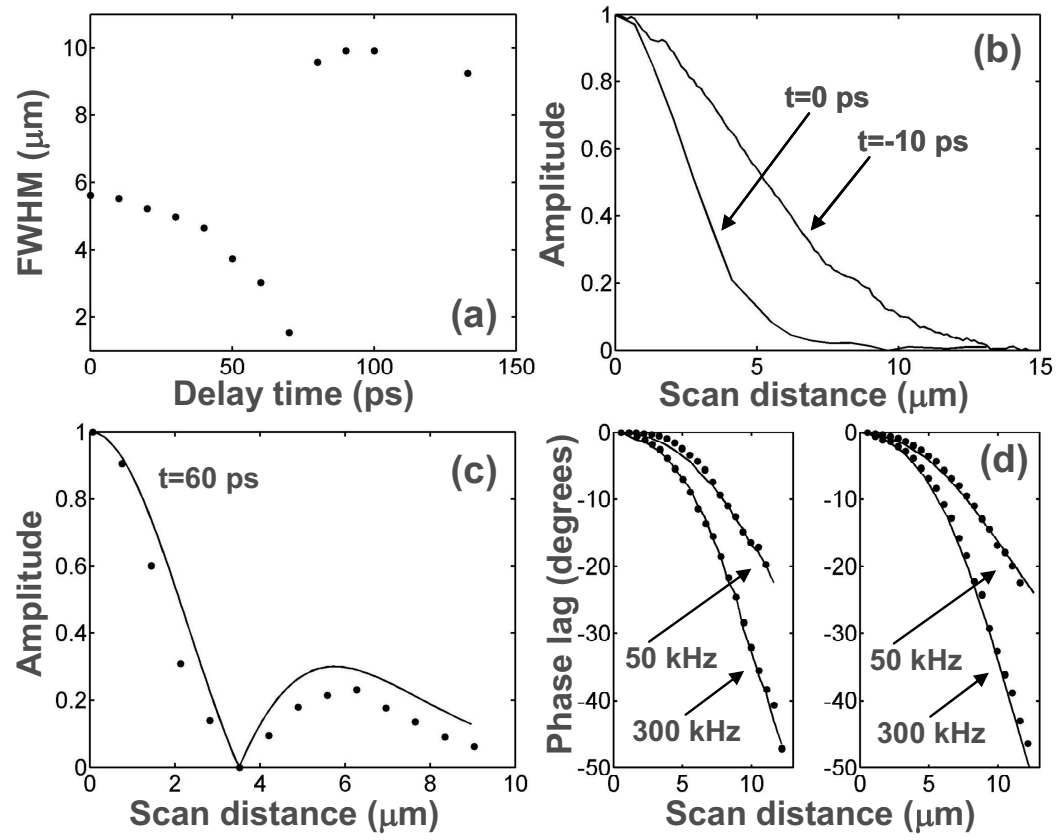

Fig. 3. Examination of the diffusion wave component. Fig. 3a gives the FWHM of the amplitude versus delay time. Fig. $3 \mathrm{~b}$ gives the amplitude profile for two different delay times. Fig. $3 \mathrm{c}$ compares the experimentally measured amplitude profile (dots) to a simulated profile (solid). Fig. 3d (left) compares the steady state phase profile for the pristine sample (dots) and the sample coated with a $30 \mathrm{~nm}$ Al film (solid). Fig. 3d (right) compares the predicted phase profile (solid) to the experimentally measured profile for the pristine sample (dots).

where $a_{t}$ and $a_{s}$ are the FWHM corresponding to the transient and diffusion wave components and $A$ represents the relative magnitude of the these two terms. (A more accurate representation of the combined amplitude response would consider the lateral variation in both the amplitude and phase of the transient and steady state components. Furthermore, while the amplitude variation of the transient component closely follows the Gaussian spatial distribution of the laser spot, the amplitude of the steady state diffusion component is only approximated using a Gaussian distribution.) At $t=0$ ps the FWHM is related to a convolution between the probe and pump profiles and serves as an upper limit to the FWHM of the pump beam profile. By measuring $a_{t}(5.8 \mu \mathrm{m})$ and $a_{s}(10 \mu \mathrm{m})$ for short and long delay times, the expression given above can be fitted to the profile for any given delay using $A$ as a fitting parameter. The results of this procedure for a delay of $60 \mathrm{ps}$ are shown in Fig. 3c. The best fit was obtained for $A=2.0$ and a sum square error of 0.31 . Note that the FWHM of the central region is smaller than the FWHM at $t=0$ ps. This clearly shows the interference between the transient and diffusion wave components.

This analysis helps explain the physical mechanism responsible for the sudden transition to steady state behavior. The initial decrease in the transient component is due to the evolution of the electron-hole plasma and diffusion of heat. However, considerable insight can be gained by considering only the electron hole plasma. The change in probe reflectivity associated with the expanding electron-hole plasma is proportional to the change in the density of photoexcited electrons. ${ }^{8}$ At $\mathrm{t}=100 \mathrm{ps}$, the electron density is approximately four times smaller than the initial density (diffusion length at $\mathrm{t}=100 \mathrm{ps}$ is $\sim 200 \mathrm{~nm}$ and the corresponding volume associated with the electron-hole plasma has increased over the initial volume by a factor of approximately four). However, from Fig. 2, the maximum change in the amplitude of the probe reflectivity at $100 \mathrm{ps}$ is approximate 30 times smaller than the contribution at $\mathrm{t}=0$. Thus, diffusion of the electron-hole plasma alone can not account for the dominance of the steady state component at $\mathrm{t}>100 \mathrm{ps}$. Recombination of the photoexcited carriers on this time scale provides an alternative explanation. The Auger recombination pathway can be neglected since the recombination time for the range of carrier concentrations used 
extends from $2 \mathrm{~ns}$ to $200 \mathrm{~ns}$. The range in recombination time $\left(\tau=1 / C N^{2}, C=5 \times 10^{-31} \mathrm{~cm}^{6} / \mathrm{s}\right)$ is estimated using the maximum carrier density range $\left(3 \times 10^{18} / \mathrm{cm}^{3}-3 \times 10^{19} / \mathrm{cm}^{3}\right) .{ }^{9}$ Surface recombination associated with defect sites ${ }^{10,11}$ is another possible pathway. The density of surface defects is expected to be high for the unpassivated sample under investigation. Studies have shown that the recombination time in unpassivated Si is of the same order as the transition time from transient to steady state behavior reported here. ${ }^{12,13}$

If indeed the electrons recombine after $\sim 100 \mathrm{ps}$, then the steady state component should be driven solely by the diffusion of heat (thermal wave). To verify this assertion a second experiment was conducted that involved coating the sample with a thin $(30 \mathrm{~nm})$ aluminum film. The aluminum film serves two purposes. First, most of the pump energy is absorbed in the aluminum film. Thus, few photoexcited carriers are created in the silicon substrate. Second, the influence of diffusing electrons is effectively screened by the aluminum film leaving only a thermal wave component. The lock-in phase responses for the pristine sample (solid) and the coated sample (dotted) for a delay time $t=133 \mathrm{ps}$ are shown on the left hand side of Fig. 3d. Two different pump modulation frequencies were used $(50 \mathrm{kHz}$ and $300 \mathrm{kHz})$. Additionally, the thermal wave was modeled taking into account the effect of the aluminum film. This model is similar to that of Reichling and Gronbeck ${ }^{14}$ and uses a Gaussian source profile. The results of this model are presented on the right hand side of Fig. 3d. The close agreement between the pristine substrate and the coated substrate as well as the close agreement between the pristine substrate and prediction confirm that after transition the steady state is to be associated with a thermal wave. It should be noted that the predicted thermal wave phase for the bare substrate and the coated sample are almost identical owing to the near parity in Si and Al thermal diffusivity. Moreover for these modulation frequencies, the thermal wavelength $(\sim 12 \mu \mathrm{m}$ at $200 \mathrm{kHz}$ for both materials $)$ is large relative to the film thickness and as a result the thermal wave penetrates a considerable distance into the Si substrate and is a function primarily of the substrate thermal properties.
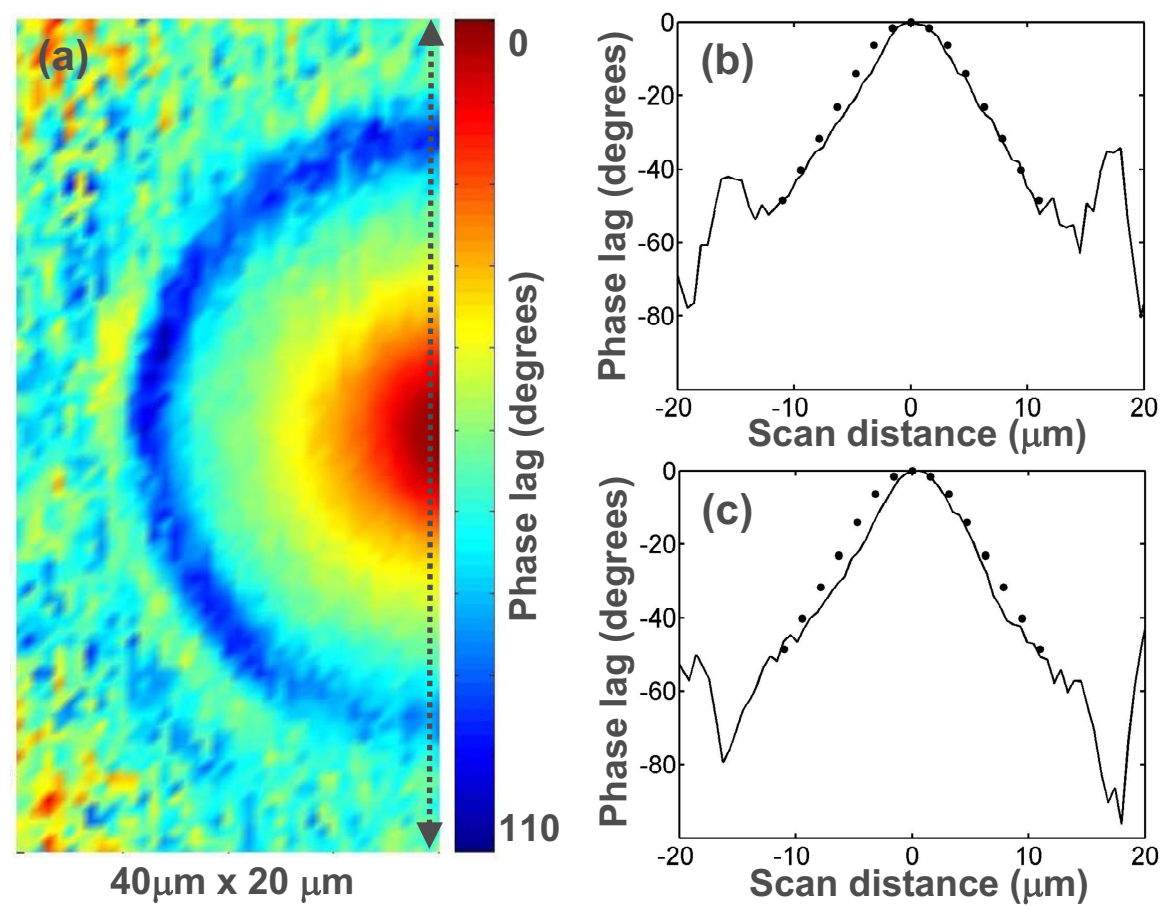

Fig. 4a. Interferometric image of thermal wave and the surface acoustic phonon pulse. Fig. 4b and 4c. Profile of the image along the superposed line for maximum positive and negative interferometric phase sensitivity.

The photoexcited electrons, in addition to being a source of thermal waves, also serve to excite coherent acoustic phonon pulses. In $\mathrm{Si}$, the photoexcitation of carriers into the conduction band followed by the fast relaxation to the band edge and subsequent phonon assisted recombination produce a localized hydrostatic stress in the material. This stress is given 
by the sum of the thermal stress $(\propto B \beta \Delta T)$ and the electronic stress arising from the hydrostatic deformation potential $\propto$ $\left(B \partial E_{g} / \partial p N\right)$, where $B$ is the bulk elastic modulus and $\beta$ is the linear thermal expansion coefficient. This stress distribution serves to launch bulk and surface acoustic phonon pulses. In the present case involving same side generation and detection, only acoustic phonon pulses that propagate along the surface of the sample are resolved.

The surface acoustic phonon pulse is imaged using a common path Sagnac interferometer to detect changes in optical phase as well as changes in probe reflectivity. ${ }^{15,16,17}$ The interferometer relies on the interference between a probe pulse that arrives before the pump (reference pulse) and a second probe pulse that arrives after the pump pulse (signal pulse). The polarity of the optical phase sensitivity of the interferometer can be changed using a $\lambda / 4$ plate. ${ }^{15}$ An interferometric phase image of the thermal wave component and the surface acoustic phonon pulse are shown in Fig. 4a. The pump modulation frequency was set to $200 \mathrm{kHz}$ to aid in the imaging of both the thermal wave and the surface acoustic phonon pulse. At this modulation frequency the thermal wave amplitude dominates the surface acoustic phonon amplitude; however, by monitoring the lock-in phase instead of the lock-in amplitude, both the thermal wave and the surface acoustic phonon pulse are imaged with comparable signal contrast. The image was formed by raster scanning the interferometric probe for a fixed pump probe delay time (3ns). The information contained in Fig. $4 \mathrm{a}$ is better understood by taking a cross section of the data along the superposed line shown on the right hand side of Fig. 4a. The data in Fig. $4 \mathrm{~b}$ and $4 \mathrm{c}$ correspond to maximum positive and negative interferometric phase sensitivity respectively. The linear variation near the center corresponds to the thermal wave component of the signal. Since this is the steady state component of the signal, the interferometer only senses changes in probe reflectivity. The low frequency detection limit $(\sim 1 \mathrm{GHz})$ of the two beam interferometer is related to path length difference of the two beams upon striking the sample. This is well above the steady state component at the pump modulation frequency $(200 \mathrm{kHz})$. The slope of this variation is the same in both figures and is directly related to the thermal diffusivity. ${ }^{18}$ The predicted variation in the thermal wave phase (dots) is also shown in Fig. $4 \mathrm{~b}$ and $4 \mathrm{c}$.

The rapid variation in the lock-in phase signal near the edges of Figs. $4 \mathrm{~b}$ and $4 \mathrm{c}$ correspond to the surface acoustic phonon pulse. The surface acoustic pulse is a transient component, and as a result the Sagnac interferometer senses changes in optical phase as well as strain induced changes in reflectivity. However, since the polarity of the surface acoustic pulse changes for positive and negative interferometric phase sensitivity, the optical phase contribution is the dominant contributor to the signal. The shape of the surface acoustic phonon wavefront is related to the symmetry of the elastic stiffness tensor and has been modeled previously. ${ }^{19}$

\subsection{Conclusion}

In conclusion, we have microscopically imaged thermal diffusion and surface acoustic phonon propagation within a single crystallite of a polycrystalline Si sample. The experimental approach holds the potential to measure the thermal diffusivity, the elastic stiffness tensor and the carrier recombination time. This approach should find wide application in the investigation of carrier and phonon transport properties in novel solar electric and optoelectronic materials.

\section{REFERENCES}

1 Thomsen C., Grahn H. T., Maris H. J., Tauc J., "Surface Generation and Detection of Phonons by Picosecond Light Pulses," Phys. Rev. B, 34, 4129 (1986).

2 Wright O. B., Perrin B., Matsuda O., Gusev V. E., "Ultrafast carrier diffusion in gallium arsenide probed with picosecond acoustic pulses,'Phys. Rev. B, 64, 081202(R) (2001).

3 von der Linde D., Kuhl J., Klingenberg H., "Raman Scattering from Nonequilibrium LO Phonons with Picosecond Resolution," Phys. Rev. Lett. 44, 1505 (1980).

4 Yoon H. W., Wake D. R., Wake J. P., Morkoc H., "Inplane Transport of photoexcited carriers in GaAs Quantum-wells," Phys. Rev. B 46, 13461 (1992).

5 Wright O. B., Gusev V. E., "Ultrafast acoustic phonon generation in gold," Physica B 220, 770 (1996). 
6 Cahill D. G., Ford W. K., Goodson K. E., Mahan G. D., Majumdar A., Maris H. J., Merlin R., Phillpot S. R. "Nanoscale thermal transport,” J. Appl. Phys. 93, 793 (2003).

7 Tachizaki T., Muroya T., Matsuda O., Sugawara Y., Hurley D. H., Wright O. B., "Scanning ultrafast Sagnac interferometry for imaging two-dimensional surface wave propagation,” Rev. Sci. Instrum. 77, 043713 (2006).

${ }^{8}$ Opsal J., Rosencwaig A., “Thermal and plasma wave depth profiling in silicon,” Appl. Phys. Lett., 47, 498 (1985).

9 V. E. Gusev, A. A. Karabutov, Laser Optoacoustics (AIP Press, New York, 1993).

${ }^{10}$ Shockley W., Read W. T., "Statistics of the Recombinations of Holes and Electrons,” Phys. Rev., 87, 835 (1952).

${ }^{11}$ Hall R. N., "Electron-Hole Recombination in Germanium,” Phys. Rev., 87, 387 (1952).

${ }^{12}$ Doany R. E., Grischkowsky D., Chi C-C., “Carrier lifetime versus ion-implantation dose in silicon on sapphire,” Appl. Phys. Lett., 50,460 (1987).

${ }^{13}$ Bambha N. K., Nighan W. L., Campbell I. H., Fauchet P. M., "Trapping time in processed polycrystalline silicon measured by picosecond time-resolved reflectivity,” J. Appl. Phys., 63, 2316 (1988).

${ }^{14}$ Reichling M., Gronbeck H., "Harmonic heat flow in isotropic layered systems and its use for thin film thermal conductivity measurements," J. Appl. Phys., 75, 1914 (1994).

${ }^{15}$ Hurley D. H., Wright O. B., "Detection of Ultrafast Phenomena Using a Modified Sagnac Interferometer,” J. Opt. Lett. 24, 1305 (1999).

${ }^{16}$ Sugawara Y., Wright O. B., Matsuda O., Takigahira M., Tanaka Y., Tamura S., Gusev V.E., "Watching ripples on crystals,” Phys. Rev. Lett. 88, 185504 (2002).

${ }^{17}$ Hurley D. H., Telschow K. L., “Simultaneous microscopic imaging of elastic and thermal anisotropy,” Phys. Rev. B (R), 71,241410 (2005).

${ }^{18}$ Maznev A. A., Hartmann J., Reichling M., “Thermal wave propagation in thin films on substrates,” J. Appl. Phys. 78, 5266 (1995).

${ }^{19}$ Farnell G. W. and Adler E. L., in Physical Acoustics, edited by W. P. Mason and R. N. Thurston (Academic, New York, 1972), Vol. 9, p. 109-166. 